\title{
The importance of comprehensive neuropsychiatric care in the postencephalitic patient
}

\author{
Jessica A Harder *,1,2 \& Timothy Y Mariano ${ }^{1,2,3}$ \\ ${ }^{1}$ Department of Psychiatry, Brigham \& Women's Hospital, Boston, MA 02115, USA \\ ${ }^{2}$ Harvard Medical School, Boston, MA, USA \\ ${ }^{3}$ Butler Hospital, Providence, RI, USA \\ *Author for correspondence: Tel.: +1 617732 6753; jaharder@bwh.harvard.edu
}

\begin{abstract}
'This piece aims to raise awareness of various approaches to comprehensively manage the neuropsychiatric sequelae of encephalitis with the hope of leading to more favorable clinical outcomes."
\end{abstract}

First draft submitted: 15 June 2018; Accepted for publication: 13 July 2018; Published online: 27 September 2018

Viral encephalitis afflicts approximately 20,000 people per year in the USA [1]. Epidemiologic data suggest that prevalence and incidence of autoimmune and viral encephalitis do not differ significantly [2], thus doubling the total encephalitis burden. After surviving encephalitis, many patients are left with significant morbidity that merits ongoing care: to treat those aspects that can benefit from rehabilitation and to prevent further decline. Acutely affected individuals can demonstrate drastic behavioral changes including aggression, profound irritability and significant disinhibition; they can also exhibit dramatic emotional and perceptual disturbances including euphoric mood and psychosis. Encephalitis patients also manifest cognitive changes, with memory and attentional impairments prominent. Patients who survive the acute illness undergo a long, slow rehabilitation toward their former selves, with considerable variability in the degree of functional, cognitive and emotional recovery achieved. There is little published guidance on how to optimize rehabilitation and many clinicians and caregivers may assume that little recovery is possible. This piece aims to raise awareness of various approaches to comprehensively manage the neuropsychiatric sequelae of encephalitis with the hope of leading to more favorable clinical outcomes.

Given the paucity of data available - and the universality with which these interventions can be applied we consider all cases of postencephalitis care as one. However, it is worth noting that the different subtypes of encephalitis can present quite differently, with etiology (e.g., infectious, autoimmune) and promptness of treating the underlying cause having an influence on prognosis, potential for rehabilitation and risk for relapse.

Rehabilitative treatment modalities should be considered in this population, as there is the potential for at least partial recovery [3]. A prospective evaluation of cognitive recovery across encephalitis subtypes showed that the majority improved over time [4]. Even in individuals with postencephalitic temporal lobe atrophy, improvements in learning and memory are possible over the long term with concentrated rehabilitation efforts [5].

A first step in the care of the postencephalitic patient is to ensure that adequate medical care is provided to identify and treat the ongoing issues. An experienced case manager with deep medical knowledge can thus be instrumental in helping patients and caregivers navigate the complexities of postencephalitis care with multiple specialists. Neurologic follow-up is especially important: although the acute illness may have subsided, related morbidity can develop over time. For example, seizures can be a particularly challenging complication; in one study of herpes simplex encephalitis (HSVE), almost one quarter of survivors developed severe epilepsy, diagnosed an average of 9 months following the acute illness [6]. Psychiatric follow-up is also critical; postencephalitis patients can demonstrate severe disturbances of affect and behavior, possibly due to amygdalar damage or changes in frontal perfusion [7]. One study of HSVE survivors found psychiatric disturbance in nearly $9 \%[6]$ and ongoing problems with functioning in HSVE survivors may be more closely associated with behavioral changes than alterations in cognition [7]. Neuropsychiatric evaluation will identify ongoing emotional (anxiety, depression, psychosis) or behavioral (agitation, aggression, disinhibition) disturbances and guide psychopharmacologic management. Appropriate medication management relies 
on the use of psychotropic agents to address each problem symptomatically, often off-label. Medications frequently utilized include benzodiazepines (agitation, sleep), antipsychotics (psychosis, agitation) and anticonvulsants (mood stabilization) [8], as well as antidepressants, stimulants and cholinesterase inhibitors [1]. Novel modalities, such as transcranial magnetic stimulation (TMS), can also be considered. Repetitive TMS is already an effective therapy for treatment-resistant depression [9], although caution must be exercised using it in patients with neurological pathology such as a lowered seizure threshold. Investigationally, TMS has been studied as a potential enhancer of cognitive performance [10], and it may assist in poststroke rehabilitation by temporarily modulating cortical plasticity [11]. A dynamic phenomenon, cortical plasticity changes across the lifespan and is altered in certain disease states [12]; it can be quantified using specific TMS protocols. TMS thus may have both diagnostic and treatment roles in future management of neurobehavioral deficits following encephalitis.

A critical component of care for the postencephalitic patient is assessment of cognitive functioning to identify areas of relative weakness and strength, and define a trajectory toward rehabilitation. Specific treatments including cognitive, speech and language, and occupational therapies can then address the areas of ongoing impairment, with compensatory strategies weighted toward areas of identified strength [3]. Comprehensive neuropsychological evaluation is the first step in defining the patient's postmorbid cognitive status, and in estimating the premorbid level of cognitive function achieved. Cognitive impairment after encephalitis is exceedingly common, with one study across encephalitis subtypes finding cognitive deficits in $94 \%$ of survivors studied [13]. The patients may demonstrate deficiencies across a number of cognitive domains, including executive functioning, memory, and language [13,14]. Cognitive rehabilitation therapy is extremely valuable, and can include teaching of compensatory strategies for problems with organization, planning, task completion and integrating or coping with multiple streams of sensory input as well as memory encoding and retrieval strategies such as verbal semantic association and visual imagery association. Patient education on the uses of assistive technology for functional support may also be integrated into such sessions. Such technologies may include: smartphones, tablets or computers with programmed reminders for tasks; electronic pill dispensers to ensure correct timing and dosing of medications; and even computer-based cognitive rehabilitation games. Limited published evidence suggests that automated reminders increase success of task completion [15] but the primary author's clinical experience suggests strong utility. Speech therapy to address residual aphasia may be necessary in some individuals, and occupational therapy can be helpful for residual apraxia/dyspraxia or functional limitations not elsewhere addressed. Medications may serve as a useful adjunct in supporting global cognitive function or executive functioning specifically. Cholinesterase inhibitors are sometimes tried in this population [1]; while neither designed nor approved for this purpose, they can have some efficacy. Medications to improve attention, concentration and task completion including stimulants, wakefulness promoting agents (modafinil, armodafinil) and other pro-dopaminergic medications (bupropion, amantadine) are also worth consideration. In areas with ready access to medical care, the above treatments may be provided by neuropsychiatrists, neuropsychologists, behavioral neurologists, physiatrists, speech and language pathologists, and occupational therapists. For patients in more remote or resource-limited settings, these treatments may be delivered by general neurologists, mental healthcare providers, and primary care physicians. In these more constrained settings, consultation with a specialist can guide the generalist in designing appropriate rehabilitation approaches and medication selection. Telehealth increases access to care - particularly for patients with reduced independence and mobility due to neurologic injury [16] and is a promising option for outpatient rehabilitation after neurologic injury. Increased availability of manualized treatments (such as cognitive behavioral therapy for inattention) may also help improve the access to care and can be delivered in a teletherapy format [17].

Finally, the recovering patient must not be considered in isolation, but in the context of their unique psychosocial situation, so that an individualized treatment plan can be developed that identifies and addresses specific areas of need. Because many patients are discharged home with a diminished level of functioning, preemptive attention to related needs is necessary to promote the patient's health and safety [18]. For example, visiting nurse services can be considered when memory impairment or executive dysfunction interfere with medication adherence or monitoring. Social services may be necessary to assist the patient with identifying and applying for disability support, in-home aid for functional abilities the patient has not regained, and transportation for those unable to drive. Adult day health centers can offer supervision for the more severely impaired (or isolated) postencephalitic patients and provide a valued respite for caregivers. Care provided informally by family or friends is another vital resource that can be proactively managed for best outcomes. For patients with memory or executive difficulties stemming from their encephalitis, managing their care alone is impractical; an involved family member can ensure greater follow-up with important medical appointments. Family can also provide critical supervision of potentially dangerous activities, 
such as driving or cooking, that may need to be limited. Given the dynamic nature of cognitive rehabilitation in these patients, the need for oversight may change over time. Thus, educating families on reasonable expectations for the patient's level of independent functioning and appropriate level of support is essential at multiple time points. This should occur not only at initiation of care, but also after subsequent neuropsychological evaluations or when other pertinent data over the course of rehabilitation indicate changes in cognitive and functional status. Finally, family members can be important partners in considering what financial resources the patient brings to bear in the case that recommended treatments are not covered by insurance.

In summary, postencephalitic patients may be left with neuropsychiatric sequelae including seizures, behavior changes, emotional disturbance, and cognitive deficits, and physicians involved in their care may not be aware of the extent to which recovery is possible. Mobilizing resources early in recovery and continuing to invest in efforts at rehabilitation is worthwhile given the potential this approach has for optimizing outcomes.

\section{Financial \& competing interests disclosure}

TY Mariano was partially supported by a 2015 Brain and Behavior Research Foundation NARSAD Young Investigator Grant and a 2017 Harvard Medical School Norman E. Zinberg Fellowship in Addiction Psychiatry Research. JA Harder was partially supported by a 2017 Daland Fellowship in Clinical Investigation. TY Mariano has consulted for Janssen Pharmaceuticals, Inc. and Ad Scientiam SAS, both unrelated to the present work. JA Harder reports no conflicts of interest. The authors have no other relevant affiliations or financial involvement with any organization or entity with a financial interest in or financial conflict with the subject matter or materials discussed in the manuscript apart from those disclosed.

No writing assistance was utilized in the production of this manuscript.

\section{References}

1 Arciniegas DB, Anderson CA. Viral encephalitis: neuropsychiatric and neurobehavioral aspects. Curr. Psychiatry Rep. 6(5), 372-379 (2004).

2 Dubey D, Pittock SJ, Kelly CR et al. Autoimmune encephalitis epidemiology and a comparison to infectious encephalitis: autoimmune encephalitis. Ann. Neurol. 83(1), 166-177 (2018).

3 Christie S, Chan V, Mollayeva T, Colantonio A. Systematic review of rehabilitation intervention outcomes of adult and paediatric patients with infectious encephalitis. BMJ Open 8(5), e015928 (2018).

4 Hokkanen L, Launes J. Cognitive recovery instead of decline after acute encephalitis: a prospective follow up study. J. Neurol. Neurosurg. Psychiatry 63(2), 222-227 (1997).

5 Martın-Monzon I, Trujillo-Pozo I, Romero RR. Functional recovery after neuropsychological rehabilitation in a case of anti-N-methyl-D-aspartate receptor encephalitis: successful results suggest neural plasticity. Adv. Res. Scientific Areas 3, 2153-2157 (2012).

6 Hjalmarsson A, Blomqvist P, Sköldenberg B. Herpes simplex encephalitis in Sweden, 1990-2001: incidence, morbidity, and mortality. Clin. Infect. Dis. 45(7), 875-880 (2007).

7 Caparros-Lefebvre D, Girard-Buttaz I, Reboul S et al. Cognitive and psychiatric impairment in herpes simplex virus encephalitis suggest involvement of the amygdalo-frontal pathways. J. Neurol. 243(3), 248-256 (1996).

8 Mohammad SS, Jones H, Hong M et al. Symptomatic treatment of children with anti-NMDAR encephalitis. Dev. Med. Child. Neurol. 58(4), 376-384 (2016).

9 Dunner DL, Aaronson ST, Sackeim HA et al. A multisite, naturalistic, observational study of transcranial magnetic stimulation for patients with pharmacoresistant major depressive disorder: durability of benefit over a 1-year follow-up period. J. Clin. Psychiatry 24, 1394-1401 (2014).

10 Luber B, Lisanby SH. Enhancement of human cognitive performance using transcranial magnetic stimulation (TMS). NeuroImage 85, 961-970 (2014).

11 Smith M-C, Stinear CM. Transcranial magnetic stimulation (TMS) in stroke: ready for clinical practice? J. Clin. Neurosci. 31, 10-14 (2016).

12 Pascual-Leone A, Freitas C, Oberman L et al. Characterizing brain cortical plasticity and network dynamics across the age-span in health and disease with TMS-EEG and TMS-fMRI. Brain Topogr. 24(3-4), 302-315 (2011).

13 Pewter SM, Williams WH, Haslam C, Kay JM. Neuropsychological and psychiatric profiles in acute encephalitis in adults. Neuropsychol. Rehabil. 17(4-5), 478-505 (2007).

14 McGrath N, Anderson NE, Croxson MC, Powell KF. Herpes simplex encephalitis treated with acyclovir: diagnosis and long term outcome. J. Neurol. Neurosurg. Psychiatry 63(3), 321-326 (1997).

15 Emslie H, Wilson BA, Quirk K, Evans JJ, Watson P. Using a paging system in the rehabilitation of encephalitic patients. Neuropsychol. Rehabil. 17(4-5), 567-581 (2007). 
16 Tenforde AS, Hefner JE, Kodish-Wachs JE, Iaccarino MA, Paganoni S. Telehealth in physical medicine and rehabilitation: a narrative review. PM R 9(5S), S51-S58 (2017).

17 Tutty S, Spangler DL, Poppleton LE, Ludman EJ, Simon GE. Evaluating the effectiveness of cognitive-behavioral teletherapy in depressed adults. Behav. Ther. 41(2), 229-236 (2010).

18 Cooper J, Kierans C, Defres S et al. Care beyond the hospital ward: understanding the socio-medical trajectory of herpes simplex virus encephalitis. BMC Health Serv. Res. 17(1), 646 (2017). 